\title{
Development and validation of an electronic FFQ to assess food intake in the Slovene population
}

\author{
Mojca Bizjak ${ }^{1, *}$, Zala Jenko-Pražnikar ${ }^{1}$ and Barbara Koroušić Seljak ${ }^{2}$ \\ ${ }^{1}$ Faculty of Health Sciences, University of Primorska, Polje 42, SI-6310 Izola, Slovenia: ${ }^{2}$ Jožef Stefan Institute, \\ Ljubljana, Slovenia
}

Submitted 30 January 2013: Final revision received 18 June 2013: Accepted 16 August 2013: First published online 8 October 2013

\begin{abstract}
Objective: To develop and validate an electronic FFQ that estimates the food and nutrient intakes, especially fat intake, in a healthy, adult population from the coastal (Mediterranean) part of Slovenia.

Design: A new electronic FFQ was developed for a survey entitled 'A multidisciplinary approach in the treatment of obesity', conducted at the University of Primorska, and validated against a $3 \mathrm{~d}$ weighed food record (FR).

Setting: Coastal region of Slovenia.

Subjects: Our study population included eighty-five healthy adults aged 25-49 years, recruited from the local coastal region of Slovenia. Intakes of food groups, macronutrients and energy, estimated by the FR and the FFQ, were compared using correlation coefficients, cross-classification and Bland-Altman plots.

Results: The mean value of most nutrient intakes tended to be higher in the FFQ compared with the FR, except for carbohydrate, fibre and energy. Regression analysis demonstrated an acceptable agreement between the FFQ and FR. The FFQ was moderately correlated with the FR $(0 \cdot 30-0 \cdot 54)$, and most of the correlations increased after energy adjustment and after de-attenuation. Relatively high (more than 70\%) proportions of participants were correctly classified into the same or an adjacent quartile. Bland-Altman analysis confirmed an acceptable level of agreement between the two methods.

Conclusions: The electronic FFQ, developed for a healthy adult Slovene population from the coastal region, was shown to be a valid tool to assess food group and nutrient intakes, especially fat intakes, and to rank individuals by their intakes within gene-nutrient studies.
\end{abstract}

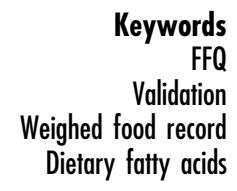

Obesity is a major contributor to the global burden of chronic disease and disability, such as insulin resistance and type 2 diabetes, CVD and certain types of cancers ${ }^{(1)}$. Worldwide, at least 300 million individuals are clinically obese $^{(2)}$, while in Slovenia $20 \cdot 8 \%$ of people aged 30 years or more are obese and $41 \cdot 0 \%$ are overweight ${ }^{(3)}$. Due to the increasing incidence of excessive weight gain and obesity, as well as the increasing costs of disease treatment, it is necessary to determine how to deal with this complex situation ${ }^{(4-7)}$. Adipose tissue, besides its role in energy storage, contributes to these co-morbidities through the secretion of pro-inflammatory mediators, which originate from adipose cells and/or infiltrated macrophages ${ }^{(8-10)}$. Identifying the molecules that link inflamed adipose tissue to complications downstream and improving our understanding of how nutrigenomics modifies adipokine secretion may facilitate prevention or amelioration of diet-related chronic diseases. Genenutrient interaction studies have the potential to reduce longstanding knowledge gaps in possible relationships between nutrients, especially dietary fat intake, genetic factors and chronic diseases ${ }^{(11,12)}$.

To understand the association between diet as a modifiable risk factor and genes, a measure of the individual's relatively short-term dietary intake is needed. Habitual dietary intake can be evaluated by different dietary methods, including food records (FR), multiple $24 \mathrm{~h}$ dietary recalls (24hDR) and FFQ. FR and $24 \mathrm{hDR}$ are accurate methods for measuring an individual's intake but they require participant motivation and literacy, and sometimes, because of their short period, certain foods and/or nutrients are lost. We assume that an FFQ over the previous month gives us a better picture of the composition of fats and, at the same time, gives us a picture of dietary habits that are important in implementing dietary interventions to prevent diet-related diseases. FFQ are also more cost-effective and easier to administer than weighed dietary records or $24 \mathrm{hDR}^{(13)}$. We reviewed a variety of questionnaires that are accessible and have been used by others ${ }^{(13-16)}$, but an FFQ developed for one 
population cannot be used in another population. An FFQ has already been developed and subsequently amended for the Slovenian population. It was used to identify the nutritional habits of the adult population of Slovenia ${ }^{(17,18)}$, but it does not incorporate marine foods, or include different species of fish or seafood, functional foods such as eggs, milk or others enriched in $n$ - 3 fatty acids, and it also does not incorporate various nuts or specific food preparations. Slovenia has different culinary regions, among which there is a traditional coastal diet, characterized as the Mediterranean diet. Therefore, because no validated FFQ was available for the population from the coastal part of Slovenia, the aim of the present study was to develop and validate a new electronic FFQ to assess the intakes of some nutrients, especially fatty acids.

\section{Experimental methods}

\section{Survey population}

A total of eighty-five healthy adults (fifty-six females and twenty-nine males) participated in the study. Inclusion criteria to participate in the present study were: (i) being from the coastal part of Slovenia; (ii) having a BMI between 19 and $35 \mathrm{~kg} / \mathrm{m}^{2}$; (iii) not taking medications for lipid disorders or anti-inflammatory drugs; (iv) not having cardiovascular, endocrine and acute or chronic inflammatory disease; (v) not having type 2 diabetes; (vi) reporting stable weight over the last 3 months; and (vii) not taking dietary supplements containing $n-3$ PUFA or fish oil. Potential participants were informed about the survey through email information from the University of Primorska and by radio, television and a local newspaper, and indicated their interest in participating by email. As required, the study was approved by the Slovenian National Medical Ethics Committee and was performed in accordance with the ethical standards laid down in the 1964 Declaration of Helsinki. All volunteers were fully informed of the procedures before written consent was obtained. All volunteers participated in the project named 'A multidisciplinary approach in the treatment of obesity', conducted at the Faculty of Health Sciences, University of Primorska, Izola.

\section{Development of FFQ}

The FFQ was implemented in electronic form within the Open Platform for Clinical Nutrition (OPEN) that is accessible through the website http://opkp.si/. At the beginning a list of relevant foods was designed. The OPEN database was updated with missing food composition data and supplemented with typical dishes. To obtain the final FFQ, the list of food items was reviewed and some changes were made. The final FFQ contained forty-five questions about 209 food items, typical dishes, food preparation and cooking methods, with portion sizes of the food items presented. For each question we created a portion size and took a picture. Portion sizes and amounts of food for culturally specific dishes were determined using traditional recipes. The portion sizes of the entrées were determined on the basis of the intake data from previous experiments, published in the Guidelines for Healthy Nutrition at Work Organizations ${ }^{(19)}$. Food was weighed according to the recipe, the dish was measured according to the guidelines and then the picture was taken. Photographs were analysed by two independent dietitians for the estimation of serving size.

The FFQ also contained questions about eating foods fortified with $n$-3 PUFA and about removing the skin from poultry and visible fat from meat. If questions related to non-specific foods e.g. the nutritional value of fresh fruit, an average value for typical fruit was used. Each food item from the fats and fatty foods group was individually listed. At the end of the listed items there was a question that allowed the participants to choose other non-listed food eaten but available in the database. Participants were asked to recall their habitual intake 1 month back in time. An FFQ design was selected using small (half a medium), medium (one portion) and large (one and a half medium) portions. The standard for medium portion size was defined by the usual portion. We calculated the usual portion (medium portion) using the formula: [(average portion for women) + (average portion for man)]/2. Questions asked in the FFQ included: 'How often in the last month did you drink milk with $3.5 \%$, $1.6 \%$ or $0.5 \%$ of milk fat - including cocoa, hot chocolate, white coffee or milk for cereals?' 'Was the milk you used fortified with $n$-3 PUFA?' 'How often in the last month did you eat baked, boiled, stewed, fried or in any other way prepared oily fish such as sardines, anchovies, mackerel, tuna or swordfish?' The eight frequency responses were: never, once per month, 2-3 times/month, 1-2 times/week, 3-4 times/week, 5-6 times/week, 1-2 times/d and 3+ times/d (Fig. 1).

\section{Dietary assessment}

FFQ were completed during the first visit to the Faculty and conducted by a trained dietitian. Participants were also given actual food portions, e.g. a serving of canned tuna, a serving of dried fruit, a serving of nuts, etc., so it was easier for them to assess the portion size of their entries. On the day of the second visit participants brought their FR. They were instructed to record their food intake for three consecutive days (two weekdays and one weekend day) the week before blood samples were taken. Where possible, participants were asked to include food labels and recipes for mixed dishes and were encouraged to avoid any alterations to their normal diet. They were taught to weigh and record all foods and beverages immediately before eating them and to weigh and describe any leftovers. In the case of meals consumed in restaurants, participants were asked to request the restaurant staff for information on the type and weight of ingredients (especially fat). All FR were checked and 


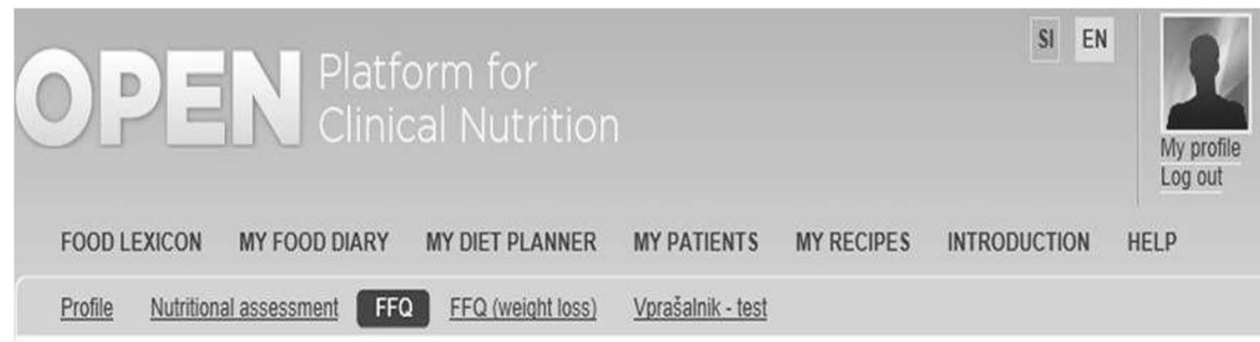

How often have you eaten fruit in the last month? Yes / No

Fresh fruit

\begin{tabular}{|l|l|l|l|}
\hline Frequency & \begin{tabular}{|l|l|}
\hline 1 per month \\
$2-3$ per month \\
$1-2$ per week \\
$3-4$ per week \\
$5-6$ per week \\
$1-2$ per day \\
$3+$ per day \\
Never
\end{tabular} \\
\hline
\end{tabular}

Fig. 1 Sample page of the FFQ, available on the website (http://opkp.si/) named OPEN (Open Platform for Clinical Nutrition), with the question and eight frequency responses, three typical quantities of food and photographs for portion size estimation

Table 1 Nutritional composition and energy value of one food unit from each food group

\begin{tabular}{|c|c|c|c|c|c|}
\hline Food group & Carbohydrate $(\mathrm{g})$ & Protein $(\mathrm{g})$ & Total fat $(\mathrm{g})$ & Energy (kJ) & Energy (kcal) \\
\hline Milk and milk products & 10 & 7 & 3 & 400 & 95 \\
\hline Vegetables & 5 & 2 & 0 & 118 & 28 \\
\hline Fruit & 15 & 0 & 0 & 250 & 60 \\
\hline Starchy foods & 15 & 2 & 0 & 300 & 70 \\
\hline Legumes & 15 & 5 & 0 & 370 & 83 \\
\hline Meat and meat products & 0 & 7 & 7 & 390 & 93 \\
\hline Fat and fatty foods & 0 & 0 & 5 & 200 & 48 \\
\hline Sugars & 10 & 0 & 0 & 170 & 40 \\
\hline
\end{tabular}

completed by dietitians if unclear descriptions or a lack of data became evident. Dietary data were analysed using the OPEN facility for food diary analysis. Data from the FFQ and FR were automatically converted into intakes of energy and nutrients, namely protein, carbohydrate, fibre, total fat, SFA, MUFA, PUFA and $n$-3 PUFA, and into food units. A food unit represents the net amount of food consumed without wastage and each food unit from every selected food group contains similar amounts of carbohydrate, protein, total fat and energy (Table 1).

\section{Food items}

We applied food composition data from the Slovenian food composition database that was analysed, compiled and indexed by the Biotechnical Faculty of Ljubljana University and the Jožef Stefan Institute ${ }^{(20)}$. Missing data were borrowed from the Souci-Fachmann-Kraut food composition database $^{(21)}$ and the National Nutrient Database for Standard Reference of the US Department of Agriculture ${ }^{(22)}$.

Food composition data applied by OPEN met the European standard for food data 'Structure and format standard' (EN 16104:2012) ${ }^{(23)}$. As OPEN has been designed for use in different countries, it can easily change the underlying food composition data with any food composition database that complies with the EuroFIR standards ${ }^{(24)}$ for data compilation and usage.

To evaluate the FR and FFQ we used analysed composition data for simple (processed) foods like fruits, vegetables, milk and milk products, bread, etc. For composite meat and fish dishes we used calculated 
composition data. We collected typical Slovenian recipes for foods/dishes that are consumed in Primorska (i.e. the coastal part of Slovenia) and calculated composition data using the OPEN online recipe calculation method ${ }^{(25)}$. This relies on the procedure that was originally recommended by INFOODS ${ }^{(26)}$ and has been acknowledged by EuroFIR $^{(27)}$. It takes the yield factors at the recipe level and the nutrient retention factors at the ingredient level. For cooked ingredients for which data are available, the yield factors for water and fat changes are taken at the ingredient level. When salted or sugared cooking water or marinating liquid is drained after the process of cooking or marinating, respectively, the method adjusts the amount of $\mathrm{Na}$ or simple sugars in the recipe by solute diffusion. As the Slovenian food composition database omits retention and yield factors, OPEN takes into account the factors published by Bognár ${ }^{(28)}$ for calculation of the composition of Slovenian recipes.

\section{Statistical metbods}

Means and standard deviations were calculated for total nutrient intakes from the FFQ and FR. We log-transformed the data to improve normality of their distribution. The validity of the FFQ was evaluated by comparing the mean nutrient intake data obtained from the FFQ with the mean nutrient intake data obtained from the FR. Pearson correlation coefficients were used to assess the association between nutrient intake estimates from the FFQ and FR, covering the same 1-month period. De-attenuated correlations were calculated to remove the within-person variability. Relative agreement between the two methods was tested by cross-classification of the nutrient score and estimation of the proportion of the participants who were classified by the two methods into the same, adjacent and extreme quartiles. Bland-Altman limits of agreement were used to evaluate the level of agreement between the two dietary methods across the range of intakes. The difference in means between the two methods was plotted against the average of the two methods for each macronutrient. Data were analysed using the statistical software package IBM SPSS Statistics19.

\section{Results}

Overall, 105 women and men participated in the validation study; twenty individuals were excluded from the present analysis as their BMI was above $35 \mathrm{~kg} / \mathrm{m}^{2}$, or because they consumed supplements containing $n-3$ PUFA or fish oil, or because they reported not living in the coastal part of Slovenia. The results presented here are based on the dietary assessment of eighty-five participants who completed the required FFQ and FR. Table 2 presents some major characteristics of the study population of the validation study, which consisted of $34 \%$ men and $66 \%$ women. Mean age was $37 \cdot 4$ years (range $25-49$ years),

\begin{tabular}{lc}
$\begin{array}{l}\text { Table } 2 \text { Characteristics of study the population: } \\
\text { healthy adults }(n \text { 85) aged } 25-49 \text { years, coastal } \\
\text { region of Slovenia }\end{array}$ \\
\hline Characteristic & Value \\
\hline Sex & \\
Male & $n 29,34 \%$ \\
Female & $n 56,66 \%$ \\
Age (years) & \\
Range & $25-49$ \\
Mean & $37 \cdot 4$ \\
SD & $6 \cdot 2$ \\
BMl (kg/m ${ }^{2}$ ) & \\
Range & $17 \cdot 5-36 \cdot 8$ \\
Mean & $25 \cdot 9$ \\
SD & $4 \cdot 5$ \\
$<25 \cdot 0$ & $n 39,46 \%$ \\
$>25 \cdot 0$ & $n 6,54 \%$ \\
Body fat (\%) & \\
Range & $5 \cdot 1-43 \cdot 7$ \\
Mean & $27 \cdot 7$ \\
SD & $9 \cdot 6$ \\
\hline
\end{tabular}

mean BMI was $27 \cdot 7 \mathrm{~kg} / \mathrm{m}^{2}$ and mean body fat was $25 \cdot 9 \%$. Based on these characteristics, the sample selected for the validation study was considered representative.

\section{Intake levels measured by FFQ compared with 3 d weighed food record}

In all participants, the mean energy intake amounted to $8640 \mathrm{~kJ}$ (2065 kcal) based on the FFQ and $8895 \mathrm{~kJ}$ $(2126 \mathrm{kcal})$ based on the FR (FFQ:FR $=0.97)$. Compared with the FR, apart from energy, carbohydrate and fibre intakes, the FFQ overestimated the intake of most macronutrients, with intake ratios obtained by the comparison of the FFQ and FR ranging from $1 \cdot 10$ for protein to 1.75 for MUFA (Table 3).

When the intake of macronutrients was expressed as a percentage of total energy intake, the overestimation by FFQ compared with FR diminished (Table 3), with ratios ranging from 1.09 for protein to 1.69 for MUFA. Stratified analyses of intake levels by age $(<35 v$. $>35$ years $)$ and BMI $\left(>25 \cdot 0 \quad v .<25 \cdot 0 \mathrm{~kg} / \mathrm{m}^{2}\right)$ showed no systemic differences in intake levels.

The mean Pearson's correlation between absolute intakes as measured by the FFQ and FR for energy intake and macronutrients was $0 \cdot 39$, ranging from $0 \cdot 27$ for MUFA to 0.52 for fibre. When intake was expressed as an energy percentage, apart from SFA, correlations increased for most macronutrients. The mean Pearson's correlation between intakes expressed as an energy percentage was $0 \cdot 43$, ranging from 0.30 for MUFA to 0.54 for fibre. De-attenuation improved the correlation coefficients for all nutrients with a mean value $0 \cdot 44$ (Table 4).

Because of our interest in the composition of dietary fat, we also expressed different fats as a fat percentage (Table 3). In this situation correlations increased for all fats and the mean Pearson's correlation between intake measured by the FFQ and FR for fats was $0 \cdot 40$, ranging from 0.32 for MUFA to 0.45 for PUFA (Table 4 ). 
Table 3 Mean daily nutrient intakes estimated by the FFQ and FR among healthy adults $(n$ 85) aged $25-49$ years, coastal region of Slovenia

\begin{tabular}{|c|c|c|c|c|c|c|}
\hline \multirow[b]{2}{*}{ Nutrient } & \multicolumn{2}{|c|}{ FFQ } & \multicolumn{2}{|c|}{$\mathrm{FR}$} & \multicolumn{2}{|c|}{ FFQ/FR } \\
\hline & Mean & SD & Mean & SD & Mean & $S D$ \\
\hline Energy (kJ) & 8640 & 2586 & 8895 & 2703 & 0.97 & 0.33 \\
\hline Energy (kcal) & 2065 & 618 & 2126 & 646 & 0.97 & 0.33 \\
\hline Protein $(\mathrm{g})$ & $89 \cdot 0$ & $33 \cdot 0$ & $87 \cdot 0$ & $30 \cdot 0$ & $1 \cdot 10$ & 0.42 \\
\hline Protein (en \%) & $17 \cdot 3$ & $3 \cdot 4$ & $16 \cdot 6$ & $4 \cdot 0$ & 1.09 & 0.27 \\
\hline Carbohydrate (g) & $199 \cdot 0$ & $65 \cdot 0$ & $252 \cdot 0$ & $89 \cdot 0$ & 0.85 & 0.34 \\
\hline Carbohydrate (en \%) & $38 \cdot 7$ & $6 \cdot 5$ & $47 \cdot 2$ & $8 \cdot 0$ & 0.84 & $0 \cdot 16$ \\
\hline Fibre $(\mathrm{g})$ & $21 \cdot 1$ & $9 \cdot 8$ & 23.0 & $13 \cdot 0$ & 0.92 & 0.44 \\
\hline Fibre (en \%) & $4 \cdot 2$ & 1.5 & $4 \cdot 3$ & $1 \cdot 8$ & 0.98 & 0.36 \\
\hline Total fat $(\mathrm{g})$ & $98 \cdot 0$ & $39 \cdot 0$ & $82 \cdot 0$ & $27 \cdot 0$ & $1 \cdot 28$ & 0.51 \\
\hline Total fat (en \%) & $42 \cdot 3$ & $6 \cdot 6$ & $34 \cdot 6$ & $6 \cdot 1$ & $1 \cdot 26$ & 0.29 \\
\hline SFA (g) & $33 \cdot 0$ & $14 \cdot 0$ & $26 \cdot 0$ & $11 \cdot 0$ & $1 \cdot 39$ & 0.62 \\
\hline SFA (en \%) & $14 \cdot 3$ & 3.0 & $11 \cdot 1$ & $2 \cdot 9$ & $1 \cdot 36$ & 0.36 \\
\hline SFA (fat \%) & 33.9 & $5 \cdot 5$ & $32 \cdot 2$ & $7 \cdot 0$ & 1.09 & 0.26 \\
\hline MUFA (q) & $35 \cdot 0$ & $15 \cdot 0$ & 23.0 & $10 \cdot 0$ & $1 \cdot 75$ & 0.00 \\
\hline MUFA (en \%) & $15 \cdot 0$ & $4 \cdot 4$ & $9 \cdot 8$ & $3 \cdot 6$ & 1.69 & $0 \cdot 71$ \\
\hline MUFA (fat \%) & $35 \cdot 1$ & $7 \cdot 4$ & $28 \cdot 2$ & $8 \cdot 5$ & $1 \cdot 24$ & 0.38 \\
\hline PUFA (g) & $11 \cdot 7$ & $4 \cdot 6$ & $11 \cdot \overline{6}$ & $5 \cdot 2$ & $1 \cdot 16$ & 0.57 \\
\hline PUFA (en \%) & $5 \cdot 1$ & $1 \cdot 3$ & 4.9 & $2 \cdot 1$ & $1 \cdot 13$ & 0.43 \\
\hline PUFA (fat \%) & $13 \cdot 1$ & $3 \cdot 2$ & $14 \cdot 3$ & $5 \cdot 9$ & 0.92 & 0.25 \\
\hline$n-3$ PUFA (g) & $1 \cdot 4$ & $0 \cdot 7$ & $1 \cdot 1$ & $0 \cdot 6$ & 1.55 & $1 \cdot 00$ \\
\hline$n$-3 PUFA (en \%) & 0.6 & $0 \cdot 2$ & 0.6 & 0.3 & 0.95 & 0.87 \\
\hline$n-3$ PUFA (fat \%) & 1.5 & $0 . \overline{8}$ & $1 \cdot 5$ & $1 \cdot 1$ & $1 \cdot 00$ & $0 \cdot 70$ \\
\hline
\end{tabular}

FR, $3 \mathrm{~d}$ weighed food record; en \%, percentage of energy intake; fat \%, percentage of total fat intake.

Table 4 Pearson correlation coefficients between daily consumption of nutrients estimated by the FFQ $v$. FR among healthy adults ( $n$ 85) aged 25-49 years, coastal region of Slovenia

\begin{tabular}{lcccc}
\hline & \multicolumn{3}{c}{ Correlation of FFQ $v$. FR } \\
\cline { 2 - 5 } Nutrient & Crude* & Energy-adjustedt & Fat-adjusted & De-attenuated \\
\hline Energy (kJ) & 0.416 & - & - & 0.488 \\
Protein (g) & 0.331 & 0.470 & - & 0.346 \\
Carbohydrate (g) & 0.412 & 0.456 & - & 0.442 \\
Fibre (g) & 0.516 & 0.537 & - & 0.579 \\
Total fat (g) & 0.364 & 0.441 & 0.395 & 0.408 \\
SFA (g) & 0.423 & 0.390 & 0.315 & 0.478 \\
MUFA (g) & 0.274 & 0.297 & 0.454 & 0.324 \\
PUFA (g) & 0.344 & 0.422 & 0.412 & 0.464 \\
$n-3$ PUFA (g) & 0.385 & 0.395 & 0.455
\end{tabular}

FR, 3 d weighed food record.

${ }^{\star}$ All Pearson correlation coefficients were significant $(P<0.01)$, except for MUFA $(P<0.05)$.

†All Pearson correlation coefficients were significant $(P<0.01)$.

$\ddagger$ All Pearson correlation coefficients were significant $(P<0 \cdot 01)$.

\section{Cross-classification analysis of nutrient intakes}

The cross-classification of daily nutrient intakes measured by the FFQ and FR is shown in Table 5 . The proportion of participants classified into exactly the same quartile varied from $29 \cdot 8 \%$ (protein) to $44 \cdot 0 \%$ (MUFA) and the mean disagreement between the two methods (extreme quartiles) was $7 \cdot 0 \%$.

\section{Bland-Altman analysis}

To illustrate the limits of agreement between the two methods, we plotted Bland-Altman scatter plots for daily intakes of energy, carbohydrate, protein, total fat, MUFA and PUFA (Fig. 2). The mean difference for energy was small and indicated that the FFQ slightly (2\%) underestimated daily energy intake (FFQ:FR =0.98); the underestimation was higher in daily carbohydrate intake $(10 \%)$. On the other hand, the mean difference for total fat indicated that the FFQ overestimated daily total fat intake (FFQ:FR $=1 \cdot 07$ ), and the overestimation was highest in daily PUFA intake (overestimation by $23 \%$ ). For energy, macronutrients and all fats, a few individuals fell outside the $95 \%$ limit of agreements and for all measurements, the mean differences were not associated with the means of the two methods, confirming an acceptable level of agreement between the two methods. Mean FFQ:FR values (95\% CI) were as follows: $0.98(95 \%$ CI $0.76,1 \cdot 20), 1.01(95 \%$ CI 0.76 , $1 \cdot 36), 0 \cdot 90(95 \%$ CI $0 \cdot 58,1 \cdot 23), 1 \cdot 07$ (95\% CI $0 \cdot 72,1 \cdot 42)$, 
Table 5 Cross-classification of nutrient intakes: percentage agreement between the FFQ and FR among healthy adults ( $n$ 85) aged 25-49 years, coastal region of Slovenia

\begin{tabular}{lccc}
\hline & \multicolumn{3}{c}{ Agreement - comparison by quartile } \\
\cline { 2 - 4 } Nutrient & Same quartile & Adjacent quartile & Misclassified* $^{*}$ \\
\hline Energy (kJ) & $34 \cdot 5$ & $46 \cdot 1$ & $8 \cdot 2$ \\
Protein (g) & $29 \cdot 8$ & $54 \cdot 1$ & $5 \cdot 9$ \\
Carbohydrate (g) & $34 \cdot 5$ & $44 \cdot 7$ & $7 \cdot 1$ \\
Fibre (g) & $36 \cdot 9$ & $42 \cdot 4$ & $7 \cdot 1$ \\
Total fat (g) & $33 \cdot 3$ & $50 \cdot 6$ & $3 \cdot 5$ \\
SFA (g) & $33 \cdot 3$ & $29 \cdot 4$ & $3 \cdot 5$ \\
MUFA (g) & $44 \cdot 0$ & $41 \cdot 2$ & $7 \cdot 1$ \\
PUFA (g) & $34 \cdot 5$ & $42 \cdot 4$ & $10 \cdot 7$ \\
$n$-3 PUFA (g) & $36 \cdot 9$ & $32 \cdot 1$ & $9 \cdot 5$ \\
\hline
\end{tabular}

FR, 3 d weighed food record.

*Being classified from one extreme category to the other extreme category.
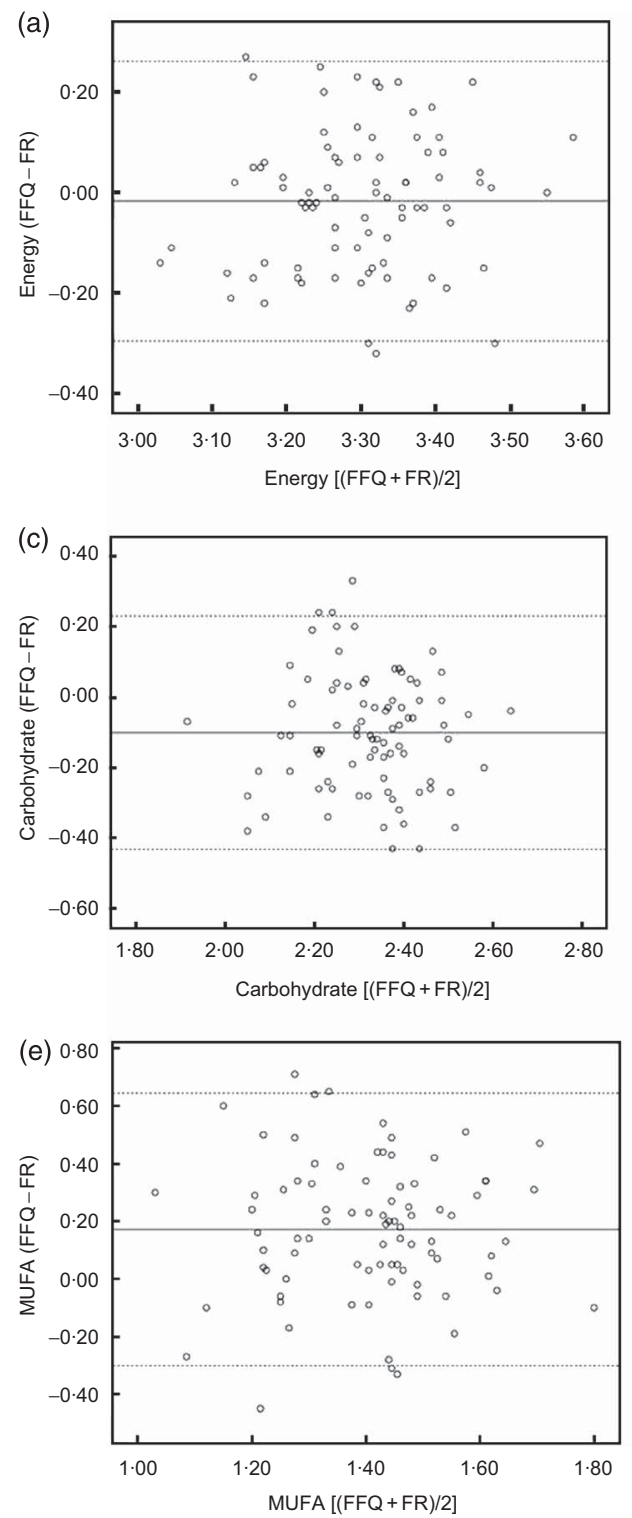

$1 \cdot 18(95 \%$ CI $0 \cdot 73,1 \cdot 63)$ and $1 \cdot 23(95 \%$ CI $0 \cdot 80,1 \cdot 66)$, respectively for energy, protein, carbohydrate, total fat, MUFA and PUFA.

\section{Dietary habits}

Table 6 presents the daily number of foods consumed for particular food groups evaluated from the FFQ and FR. Compared with the FR, apart from starchy foods, the FFQ overestimated the intake of most daily food units (Table 6). The mean Pearson's correlation between the number of units, obtained by comparison of the FFQ with the FR for food groups, was $0 \cdot 35$, ranging from $0 \cdot 18$ for vegetables to $0 \cdot 60$ for legumes.
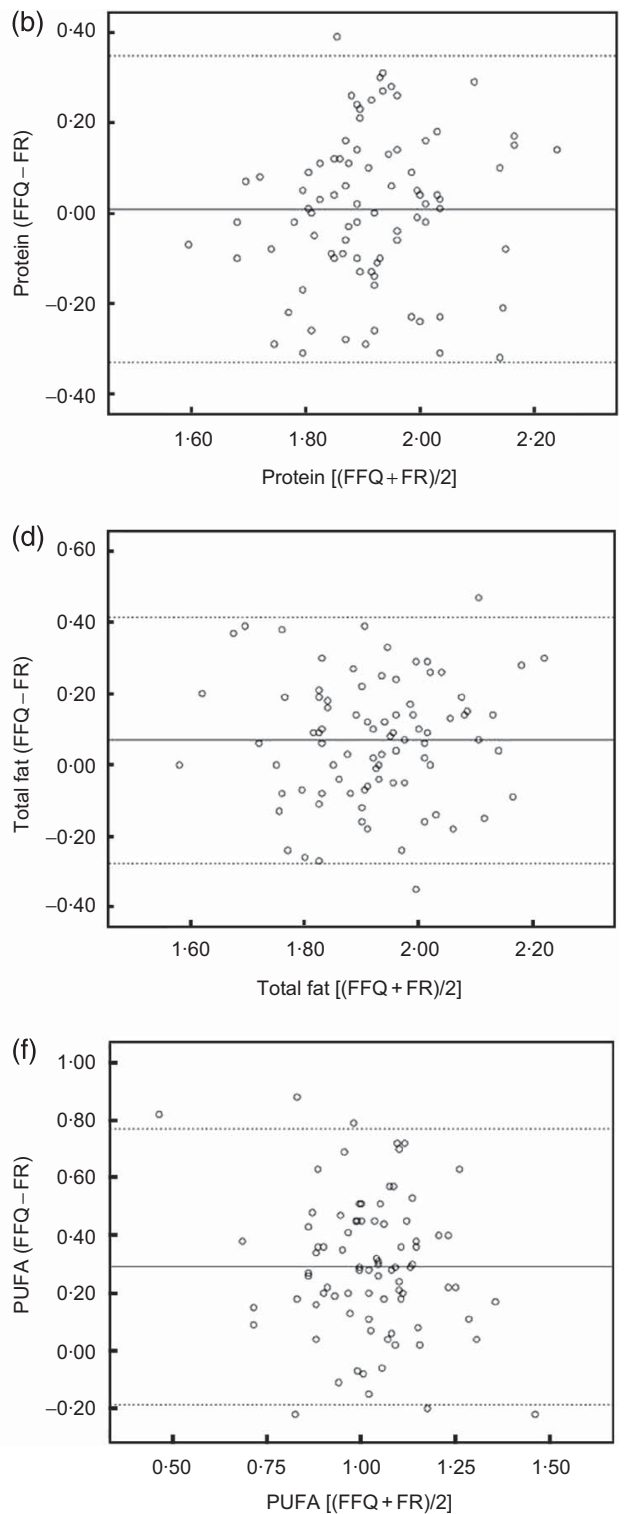

Fig. 2 Bland-Altman plots assessing the agreement between the FFQ and the $3 \mathrm{~d}$ weighed food record (FR) in estimating the intakes of (a) energy, (b) protein, (c) carbohydrate, (d) total fat, (e) MUFA and (f) PUFA among healthy adults ( $n$ 85) aged 25-49 years, coastal region of Slovenia. The difference in intake between the two methods (FFQ - FR) is plotted as a function of the mean intake of the two methods [(FFQ + FR)/2]; the solid line represents the mean difference, while the dashed lines represent the $95 \%$ limits of agreement. Data were log-transformed to improve normality of their distribution 
Table 6 Mean number of daily food units of intake evaluated from the FFQ and FR among healthy adults ( $n$ 85) aged 25-49 years, coastal region of Slovenia

\begin{tabular}{|c|c|c|c|c|c|c|}
\hline \multirow[b]{2}{*}{ Food group* } & \multicolumn{2}{|c|}{ FFQ } & \multicolumn{2}{|c|}{ FR } & \multicolumn{2}{|c|}{ Correlation of FFQ $v . F F$} \\
\hline & Mean & SD & Mean & SD & $r$ & $P$ \\
\hline Milk and milk products & $3 \cdot 17$ & $1 \cdot 67$ & $2 \cdot 71$ & $1 \cdot 48$ & 0.272 & 0.012 \\
\hline Vegetables & $2 \cdot 18$ & $1 \cdot 36$ & $1 \cdot 88$ & 1.06 & $0 \cdot 183$ & 0.054 \\
\hline Fruit & $2 \cdot 33$ & 1.53 & $2 \cdot 17$ & $1 \cdot 75$ & 0.528 & 0.000 \\
\hline Starchy foods & 4.53 & $2 \cdot 26$ & $8 \cdot 60$ & $3 \cdot 45$ & 0.304 & 0.005 \\
\hline Legumes & 0.99 & 0.68 & 0.54 & 0.32 & 0.599 & 0.000 \\
\hline Meat and meat products & $5 \cdot 23$ & $2 \cdot 80$ & $4 \cdot 07$ & $2 \cdot 27$ & 0.426 & 0.000 \\
\hline Fat and fatty foods & $12 \cdot 39$ & $5 \cdot 34$ & $9 \cdot 67$ & 3.95 & 0.289 & 0.008 \\
\hline Sugars & $5 \cdot 56$ & $3 \cdot 12$ & $4 \cdot 45$ & $2 \cdot 39$ & 0.275 & 0.012 \\
\hline Olive oil (g) & $17 \cdot 48$ & $13 \cdot 98$ & $10 \cdot 15$ & $6 \cdot 54$ & 0.304 & 0.005 \\
\hline
\end{tabular}

FR, 3 d weighed food record.

*Values are expressed as number of food units from each group, except for olive oil, expressed in grams.

\section{Discussion}

The present study showed that the electronic FFQ developed is a valid instrument for assessing food intake in adults living in the coastal part of Slovenia. The FFQ had a moderate relative validity (varying from 0.3 to 0.5 ) for most macronutrients and food groups. The high proportion of participants classified into the same or adjacent quartiles (from $70 \%$ to $85 \%$ ) showed that our FFQ was capable of ranking an individual's absolute nutrient intakes according to the FR. Also the Bland-Altman plots illustrated an acceptable level of agreement between the two methods.

In the present study eighty-five men and women participated; this was similar to a large number of previous studies $^{(15,16,29-31)}$. The number of food items in our FFQ is 209. According to Cade et al. ${ }^{(32)}$, the optimum number of food items is about 100, but in our opinion a high number was necessary to obtain a more accurate report of foods eaten due to the objectives of gene-nutrient interactions and an intervention protocol for targeting obesity in our survey. Slovenia is also at the junction of the Mediterranean, Alpine and Pannonian geographical borders and there is a great variability of food consumption. In the coastal part of Slovenia some traditional Mediterranean practices have been lost or mingled with the practices of immigrants from other parts of Slovenia; therefore we added the possibility of food selection from a database which actually caused a further increase in the number of food items. This proved useful in the case of seasonal dishes, as for example almost half of the participants chose the roasted chestnuts food item from the database.

The association between an FFQ and comparative methods is usually assessed by correlation coefficients ${ }^{(13)}$, and as a result of various measurement errors in each dietary assessment method, the observed correlation coefficients provide a measure of relative validity. We found significant and moderate crude correlations between the FFQ and FR for food groups and macronutrients, and similar correlation coefficients have been reported by previous studies ${ }^{(16,33-39)}$. When intake was expressed as an energy percentage, there was good agreement in intake levels between the FFQ and FR, corroborating the notion that FFQ generally perform better if macronutrient intake is expressed as an energy percentage $^{(40)}$. Similarly, our FFQ was able to accurately estimate dietary fat composition when the intake of fat types was expressed as a percentage of total fat intake. Also the correlations between the FFQ and FR, except for SFA, improved when adjusted for energy intake and fat intake, similar to the absolute intake levels, as has been reported in previous studies ${ }^{(13)}$. In contrast to our work, the studies of Deghgan et al. and Xia et al. showed no improvement in correlations for most nutrients after energy adjustment ${ }^{(33,41)}$. De-attenuated correlations are in most cases higher than crude correlations. Our study showed that for all nutrients, de-attenuation improved correlations. As in the case of a few previous validation studies $^{(16,33,34,41)}$, our FFQ showed that a relatively high proportion of participants were correctly classified into the same or adjacent categories and only a small number of misclassifications were obtained. This illustrated that our FFQ was capable of ranking individual intakes in accordance with the FR for most of the nutrient components.

We used the Bland-Altman method to assess the bias and limits of agreement, and showed that the estimates of energy and macronutrient intakes obtained by the two methods were comparable. The distribution of points within the limits of agreement suggested that the FFQ and FR methods were comparable, although the mean of the differences indicated that the FFQ slightly underestimated energy and carbohydrate and overestimated protein and different types of fat. Limits of agreement ranged from $76 \%$ to $120 \%$ for energy and from 58\% to $123 \%$ for carbohydrate. In the study of Ambrosini et al. ${ }^{(42)}$, limits of agreement between $50 \%$ and $200 \%$ were considered suitable and clinically acceptable. Limits of agreement equal to $50 \%$ to $200 \%$ indicate that $95 \%$ of all participants' FFQ estimates were between half and two times their FR 
estimates. In addition, limits of agreements from our study were narrower than those reported by Ambrosini et $a l^{(42)}$ or by MacIntyre et $a l .{ }^{(43)}$, but wider than those reported by Jackson et $a l^{(36)}$. However, we think that the limits of agreements from our study are clinically acceptable and that the developed FFQ could be applied in the larger study.

In the present study the average nutrient values were lower for FR data compared with the average values of the FFQ, except for carbohydrate and fibre. Most daily intake values of nutrients and food groups from both measurement systems point out unhealthy dietary habits: high total fat and SFA intakes, and low intakes of vegetables and fruit. We assume that the major reason for the low consumption of vegetables and fruit was the season, as the survey took place in winter. But in any case, it is well known that a low fruit and vegetable consumption increases the consumption of energy-rich foods, reduces essential micronutrient intake, and consequently reduces health benefits and increases weight gain ${ }^{(44,45)}$. Thus, this result can also explain the high prevalence of obesity among the Slovene population ${ }^{(2)}$.

In Slovenia the validation of an FFQ had not been undertaken, so the present study is the first that has developed and validated an FFQ in the coastal part of Slovenia. The methods used in our study, including selection of the population, sample size, the standard process of FFQ development and statistical approaches, were consistent with commonly accepted practices. The standard methods of data collection, using coloured food photographs to produce reliable estimates of habitual intake, and completion of the FR and FFQ with the help of dietitians, make the FFQ a valid tool for measuring individual food intakes.

The present study had limitations. Biomarkers measuring dietary fat intake were not collected. The relative low correlations could also be due to the limitations of our reference method of a $3 \mathrm{~d}$ weighed FR because Molag et $a l^{(46)}$ showed that correlations were higher for studies comparing FFQ with a reference method of eight to fourteen dietary record days, compared with one to seven record days. Therefore, future validation studies should consider the use of biomarkers and at least an $8 \mathrm{~d}$ FR for better agreement between methods.

The FFQ will be a valid tool for estimation of nutrient intakes, especially the intakes of fatty acids, being important for gene-nutrient studies, and the comparison of nutrient intakes with recommended intakes, both important in implementing dietary interventions for the prevention of diet-related diseases. The elaboration of an electronic FFQ also provides the opportunity to extend the food database, particularly the data on some fatty acids, which gives us a large scale and upgrades options for automatic nutrient calculations.

In conclusion, the new electronic FFQ had moderate relative validity and can be used in nutritional studies for the assessment of food groups and nutrients, especially fat intakes, among Slovenian adults from the coastal part of Slovenia. Besides, this FFQ is capable of ranking individuals by their intakes in gene-nutrient studies.

\section{Acknowledgements}

Sources of funding: Funding was received from the Faculty of Health Sciences, University of Primorska for the project entitled 'A multidisciplinary approach in the treatment of obesity'; from the Slovenian Research Agency (Research Projects J3-4211); and from the European Regional Development Fund, Cross-Border Cooperation Italy-Slovenia Programme 2007-2013 (EU strategic project TRANS2CARE). The University of Primorska, the Slovenian Research Agency and the European Regional Development Fund had no role in the design, analysis or writing of this article. Conflict of interest: The authors certify that there is no conflict of interest with any financial organization regarding the material discussed in the manuscript. Authors' contributions: M.B. designed the study, the questionnaire and the food record; B.K.-S. developed an electronic version of the questionnaire and applied the food composition data; Z.J.-P. and M.B. analysed the data and prepared the results; M.B., B.K.-S. and Z.J.-P. interpreted the data and contributed in writing the manuscript. All authors made substantial contributions and gave final approval of the preliminary versions, drafting and final version. All authors met the criteria for authorship as established by the International Committee of Medical Journal Editors and believe that the paper represents honest work. Acknowledgements: The authors would like to thank all the individuals who volunteered to participate in the study, and Tamara Poklar Vatovec and Tadeja Jakus for their help in analysing the data from food records.

\section{References}

1. Pi-Sunyer FX (2002) The obesity epidemic: pathophysiology and consequences of obesity. Obes Res 10, Suppl. 2, 97S-104S.

2. World Health Organization (2013) Obesity and overweight: Fact sheet no. 311. http://www.who.int/mediacentre/ factsheets/fs311/en/index.html (accessed March 2013).

3. World Health Organization (2010) Welcome to the Global Health Observatory. http://apps.who.int/ghodata (accessed March 2012).

4. Wyatt SB, Winters KP \& Dubbert PM (2006) Overweight and obesity: prevalence, consequences, and causes of a growing public health problem. Am J Med Sci 331, 166-174.

5. Poirier P, Giles TD, Bray GA et al. (2006) Obesity and cardiovascular disease: pathophysiology, evaluation, and effect of weight loss. Arterioscler Thromb Vasc Biol 26, 968-976.

6. Ramel A, Pumberger C, Martinéz AJ et al. (2009) Cardiovascular risk factors in young, overweight, and obese European adults and associations with physical activity and omega-3 index. Nutr Res 29, 305-312. 
7. Nawaz H \& Katz DL (2001) American College of Preventive Medicine Practice Policy Statement: weight management counselling of overweight adults. Am J Prev Med 21, 73-78.

8. Gustafson B (2010) Adipose tissue, inflammation and atherosclerosis. J Atheroscler Thromb 17, 332-341.

9. Wang Z \& Nakayama T (2010) Inflammation, a link between obesity and cardiovascular disease. Mediators Inflamm 2010, 535918.

10. Blüher M (2009) Adipose tissue dysfunction in obesity. Exp Clin Endocrinol Diabetes 117, 241-250.

11. Smith CE \& Ordovás JM (2010) Fatty acid interactions with genetic polymorphisms for cardiovascular disease. Curr Opin Clin Nutr Metab Care 13, 139-144.

12. Stryjecki C \& Mutch DM (2011) Fatty acid-gene interactions, adipokines and obesity. Eur J Clin Nutr 65, 285-297.

13. Willett WC (1998) Nutritional Epidemiology. New York: Oxford University Press.

14. Willett WC, Sampson L, Stampfer MJ et al. (1985) Reproducibility and validity of a semiquantitative food frequency questionnaire. Am J Epidemiol 122, 51-65.

15. Swierk M, Williams PG, Wilcox J et al. (2011) Validation of an Australian electronic food frequency questionnaire to measure polyunsaturated fatty acid intake. Nutrition $\mathbf{2 7}$, 641-646.

16. Eng JY \& Moy FM (2011) Validation of a food frequency questionnaire to assess dietary cholesterol, total fat and different types of fat intakes among Malay adults. Asia Pac J Clin Nutr 20, 639-645.

17. Koch V (1997) Nutritional habits of Slovenian adults in health protection aspect. PhD Thesis, University of Ljubljana (in Slovene).

18. Gabrijelčič Blenkuš M \& Lavtar D (2009) Nutritional habits research. In Nutritional Habits of Slovenian Adults in Health Protection Aspect, pp. 26-42 [M Gabrijelčič Blenkuš, editor]. Ljubljana: Public Health Institute, Slovenia (in Slovene).

19. Pokorn D, Mavčec Zakotnik J, Močnik Bolčina U et al. (2008) Smernice zdravega prebranjevanja delavcev $v$ delounih organizacijah (Guidelines for Healthy Nutrition at Work Organizations). Ljubljana: Ministry of Health.

20. Golob T, Stibilj V, Žlender B et al. (2006) Slovenian Food Composition Tables - Meat and Meat Products. Ljubljana: University of Ljubljana, Biotechnical Faculty (in Slovene).

21. Souci SW, Fachmann W \& Kraut H (2008) Food Composition \& Nutrition Tables, 7th ed. Stuttgart: MedPharm Scientific Publishers.

22. US Department of Agriculture (2011) National Nutrient Database for Standard Reference. http://www.ars.usda.gov/ Services/docs.htm?docid=8964 (accessed November 2012).

23. CEN European Standard (2013) Food data. Structure and format standard (EN 16104:2012).

24. Pakkala H, Christensen T, de Victoria IM et al. (2010) Harmonised information exchange between decentralised food composition database systems. Eur J Clin Nutr 64, 58-63.

25. Koroušić Seljak B, Stibilj S, Pograjc L et al. (2013) Food composition databases for effective quality nutritional care. Food Chem 140, 553-561.

26. Greenfield H \& Southgate DAT (2003) Food Composition Data: Production, Management, and Use, 2nd ed. Rome: FAO.

27. Reinivuo H \& Laitinen K (2007) Proposal for the harmonization of recipe calculation procedures, WP2.2 Composite Foods, EuroFIR technical report D2.2.12/M2.2.4. http:// www.langual.org/Download/RecipeCalculation/Final_recipe_ calc_harmonisation.pdf (accessed September 2013).

28. Bognár A (2002) Tables on Weight Yield of Food and Retention Factors of Food Constituents for the Calculation of Nutrient Composition of Cooked Foods (Dishes). Karlsruhe: Bundesforschungsanstalt für Ernährung.
29. Ahn Y, Kwon E, Shim JE et al. (2007) Validation and reproducibility of food frequency questionnaire for Korean genome epidemiologic study. Eur J Clin Nutr 61, 1435-1441.

30. Patterson AC, Hogg RC, Kishi DM et al. (2012) Biomarker and dietary validation of a Canadian food frequency questionnaire to measure eicosapentaenoic and docosahexaenoic acid intakes from whole food, functional food, and nutraceutical sources. I Acad Nutr Diet 112, 1005-1014.

31. Sullivan BL, Brown J, Williams PG et al. (2008) Dietary validation of a new Australian food-frequency questionnaire that estimates long-chain $n-3$ polyunsaturated fatty acids. BrJ Nutr 99, 660-666.

32. Cade J, Thompson R, Burley V et al. (2002) Development, validation and utilisation of food-frequency questionnaires - a review. Public Health Nutr 5, 567-587.

33. Dehghan M, Del Cerro S, Zhang X et al. (2012) Validation of a semi-quantitative food frequency questionnaire for Argentinean adults. PLoS One 7, e37958.

34. Van Dongen MC, Lentjes MA, Wijckmans NE et al. (2011) Validation of a food-frequency questionnaire for Flemish and Italian-native subjects in Belgium: the IMMIDIET study. Nutrition 27, 302-309.

35. Schröder H, Covas MI, Marrugat J et al. (2001) Use of a three-day estimated food record, a 72-hour recall and a food-frequency questionnaire for dietary assessment in a Mediterranean Spanish population. Clin Nutr 20, 429-437.

36. Jackson MD, Walker SP, Younger NM et al. (2011) Use of a food frequency questionnaire to assess diets of Jamaican adults: validation and correlation with biomarkers. Nutr J 10, 28 .

37. Jackson MD, Motswagole BS, Kwape LD et al. (2012) Validation and reproducibility of an FFQ for use among adults in Botswana. Public Health Nutr (Epublication ahead of print version).

38. Yang M, Wang Y, Davis CG et al. (2012) Validation of an FFQ to assess short-term antioxidant intake against $30 \mathrm{~d}$ food records and plasma biomarkers. Public Health Nutr (Epublication ahead of print version)

39. Dehghan M, Martinez S, Zhang X et al. (2013) Relative validity of an FFQ to estimate daily food and nutrient intakes for Chilean adults. Public Health Nutr 16, 1782-1788.

40. Subar AF, Thompson FE, Kipnis V et al. (2001) Comparative validation of the Block, Willett, and National Cancer Institute food frequency questionnaires. Am J Epidemiol 154, 1089-1099.

41. Xia W, Sun C, Zhang L et al. (2011) Reproducibility and relative validity of a food frequency questionnaire developed for female adolescents in Suihua, North China. PLoS One 6 , e19656.

42. Ambrosini GL, van Roosbroeck SA, Mackerras D et al. (2003) The reliability of ten-year dietary recall: implications for cancer research. I Nutr 133, 2663-2668.

43. MacIntyre UE, Venter CS \& Vorster HH (2001) A culturesensitive quantitative food frequency questionnaire used in an African population: 2. Relative validation by 7-day weighed records and biomarkers. Public Health Nutr 4 , 63-71.

44. Vioque J, Weinbrenner T, Castelló A et al. (2008) Intake of fruits and vegetables in relation to 10-year weight gain among Spanish adults. Obesity (Silver Spring) 16, 664-670.

45. Sartorelli DS, Franco LJ \& Cardoso MA (2008) High intake of fruits and vegetables predicts weight loss in Brazilian overweight adults. Nutr Res 28, 233-238.

46. Molag ML, de Vries JH, Ocké MC et al. (2007) Design characteristics of food frequency questionnaires in relation to their validity. Am J Epidemiol 166, 1468-1478. 